\title{
Association between polycyclic aromatic hydrocarbons exposure and bone turnover in adults
}

\author{
Yuan-Yuei Chen ${ }^{1,2,6}$, Tung-Wei Kao ${ }^{2,3,4}$, Chung-Ching Wang' ${ }^{2}$, Chen-Jung Wu ${ }^{2,3,5}$, Yi-Chao Zhou ${ }^{2}$ and \\ Wei-Liang Chen ${ }^{2,3}$
}

${ }^{1}$ Department of Internal Medicine, Tri-Service General Hospital Songshan Branch, ${ }^{2}$ Division of Family Medicine, Department of Family and Community Medicine, Tri-Service General Hospital, ${ }^{3}$ Division of Geriatric Medicine, Department of Family and Community Medicine, Tri-Service General Hospital, School of Medicine, National Defense Medical Center, Taipei, Taiwan, Republic of China, ${ }^{4} \mathrm{Graduate}$ Institute of Clinical Medical, College of Medicine, National Taiwan University, Taipei, Taiwan, Republic of China, ${ }^{5}$ Division of Family Medicine, Department of Community Medicine, Taoyuan Armed Forces General Hospital, Taoyuan, Taiwan, Republic of China, and ${ }^{6}$ Department of General Medicine, Tri-Service General Hospital, School of Medicine, National Defense Medical Center, Taipei, Taiwan, Republic of China

Correspondence should be addressed to W-L Chen Email weiliang0508@gmail.com

\section{Abstract}

Background: Cigarette smoking is a risk factor of osteoporosis and bone fracture. Tobacco smoke contains several polycyclic aromatic hydrocarbons. Thus, we hypothesized that environmental polycyclic aromatic hydrocarbon exposure is associated with bone loss and fracture risk. The present study examined the association between polycyclic aromatic hydrocarbon exposure and bone turnover in the general adult population.

Methods: A total of 1408 eligible participants from the National Health and Nutrition Examination Survey (NHANES 2001-2006) were included in this cross-sectional analysis. The levels of urinary N-telopeptide and serum bonespecific alkaline phosphatase, which are biomarkers of bone resorption and formation, respectively, were assessed. Meanwhile, polycyclic aromatic hydrocarbon exposure was evaluated using the concentrations of urinary polycyclic aromatic hydrocarbon metabolites. The association between polycyclic aromatic hydrocarbon exposures and $\mathrm{N}$-telopeptide, and bone-specific alkaline phosphatase levels was assessed using a multivariate linear regression model.

Results: All polycyclic aromatic hydrocarbon metabolites except 3-phenanthrene were significantly associated with increased $\mathrm{N}$-telopeptide levels $(P<0.05)$ after adjustment of relevant covariables. However, no significant relationship was observed between polycyclic aromatic hydrocarbon metabolites and bone-specific alkaline phosphatase levels. This relationship remained significant after the participants were assessed according to $\operatorname{sex}(P<0.05)$. Additionally, all polycyclic aromatic hydrocarbon metabolites showed a positive association with $\mathrm{N}$-telopeptide levels in participants aged $<60$ years $(P<0.05)$.

Conclusion: Polycyclic aromatic hydrocarbon exposure is associated with increased bone resorption among the general adult population in the United States. Further studies must assess the potential mechanisms associated with the adverse effects of polycyclic aromatic hydrocarbon exposure on bone loss.

European Journal of Endocrinology

(2020) 182, 333-341
Published by Bioscientifica Ltd. 


\section{Introduction}

Polycyclic aromatic hydrocarbons are organic compounds with multiple aromatic rings of only carbon and hydrogen (1). These pollutants are generated via the incomplete combustion of organic matter from either natural or synthetic sources and are widely present in various environment matrices, such as air, soil, and water. In the general population, exposure mainly occurs via inhalation of contaminated air and consumption of contaminated food and water (2). In particular, high exposure can occur via cigarette smoking. polycyclic aromatic hydrocarbon exposure causes various systemic diseases, including cancer $(3,4)$, obstructive lung diseases (5), metabolic syndrome (6), and cardiovascular diseases $(7,8)$.

The bone is a dynamic tissue that constantly undergoes remodeling throughout life (9); this is caused by the coupling of bone formation and resorption at various rates balancing bone turnover (10). Epidemiological studies have reported that cigarette smoking contributes to decreased bone mass and osteoporosis $(11,12,13)$. Polycyclic aromatic hydrocarbon is a component of cigarette smoke that decreases bone mechanical properties, leading to bone loss (14).

To the best of our knowledge, no study has examined the relationship between polycyclic aromatic hydrocarbon exposure and bone loss in the human population. Thus, the present study investigated the associations between urinary polycyclic aromatic hydrocarbon metabolite and biomarkers of bone turnover in a cross-sectional analysis of a nationally representative general adult sample in the United States.

\section{Methods}

\section{Study design}

The National Health and Nutrition Examination Survey (NHANES) is a cross-sectional survey on a nationally representative sample that collects demographic, clinical, behavioral, dietary, social, and laboratory data about the health and nutritional status of non-institutionalized individuals in the United States. The National Center of Health Statistics (NCHS) Ethics Review Board approved the study. All participants were requested to sign an informed consent form before the initiation of the study.

\section{Participants}

Initially, adults aged $\geq 20$ years from the NHANES 20012006 were included in the study. After excluding those with missing information such as demographic and laboratory data, 1408 eligible participants were enrolled for further analysis.

\section{Assessment of bone turnover biomarker levels}

$\mathrm{N}$-telopeptide is a biomarker of bone resorption, which is a telopeptide used to evaluate the rate of bone turnover (15). N-telopeptide can be measured in the urine or blood (16). In NHANES, analysis of urinary N-telopeptide levels was performed using a competitive-inhibition enzymelinked solid-phase immunosorbent assay (Osteomark; NHANES 1999-2001) or the Vitros ECi competitive assay (NHANES 2002). Bone-specific alkaline phosphatase is synthesized by osteoblasts and is involved in the calcification of bone matrix. Moreover, it is a highly specific marker of bone formation. In this study, the bone-specific alkaline phosphatase level in the blood samples was measured using the Beckman Access Ostase assay (Beckman Coulter, LaBrea, CA, USA). The reference range for N-telopeptide was $14-87 \mathrm{nM} \mathrm{BCE} / \mathrm{mM}$ in adult males and 5-65 nM BCE/mM in premenopausal females. The intra- and inter-assay coefficients of variation were 7.9 and $10.9 \%$, respectively. The reference range for bonespecific alkaline phosphatase was 3.7-20.9 $\mu \mathrm{g} / \mathrm{L}$ in adult males and $2.9-14.5 \mu \mathrm{g} / \mathrm{L}$ in premenopausal females. The intra- and inter-assay coefficients of variation were 4.7 and $9.1 \%$, respectively.

\section{Level of urinary polycyclic aromatic hydrocarbons}

Urine samples were collected by experienced technicians and were stored at $-20^{\circ} \mathrm{C}$ according to laboratory/ medical procedures. Urinary metabolites were quantified by capillary gas chromatography combined with highresolution mass spectrometry. Details regarding the procedure are available on the NHANES website (17). The urinary hydroxylated polycyclic aromatic hydrocarbon metabolites included 1-naphthalene, 2-naphthalene, 2-fluorene, 3-fluorene, 1-phenanthrene, 2-phenanthrene, 3-phenanthrene, and 1-pyrene.

\section{Measurement of covariates}

For causal inferences from observational data, it was important to elucidate confounding, but it was not easy to recognize. A confounder was a factor that was associated with the exposure and with the outcome and was not in the causal path between the exposure and outcome. In Fig. 1, we demonstrated our Directed 
Acyclic Graph (DAG) analysis of the study based on the correlations and previous evidences. Factors of interest, such as demographic information, cigarette use, and medical histories, were assessed using the respective questionnaires. The patients were classified according to race: non-Hispanic white and others. The question 'have you ever smoked cigarettes?' was used to assess cigarette use. Patients with medical histories were defined as participants who had been diagnosed with congestive heart failure (CHF), coronary heart disease (CHD), angina, heart attack, and cancer. Laboratory data were collected using the respective standard procedures. Cotinine is the predominant metabolite of nicotine that can be used as a marker of both active smoking and environmental exposure. It is generally preferred over nicotine for such assessments due to its substantially longer half-life. Serum cotinine level was assessed using isotope dilution HPLC/atmospheric pressure chemical ionization tandem mass spectrometry. C-reactive protein (CRP) level was measured using latex-enhanced nephelometry with an automated Behring Nephelometer Analyzer System (Behring Diagnostics, Inc, Somerville, NJ, USA). Serum albumin, total calcium, and glucose levels were assessed using the Beckman Synchron LX20. All data about specimen collection are available on the NHANES dataset.

\section{Statistical analysis}

The associations between polycyclic aromatic hydrocarbons and N-telopeptide and bone-specific alkaline phosphatase were evaluated using a linear

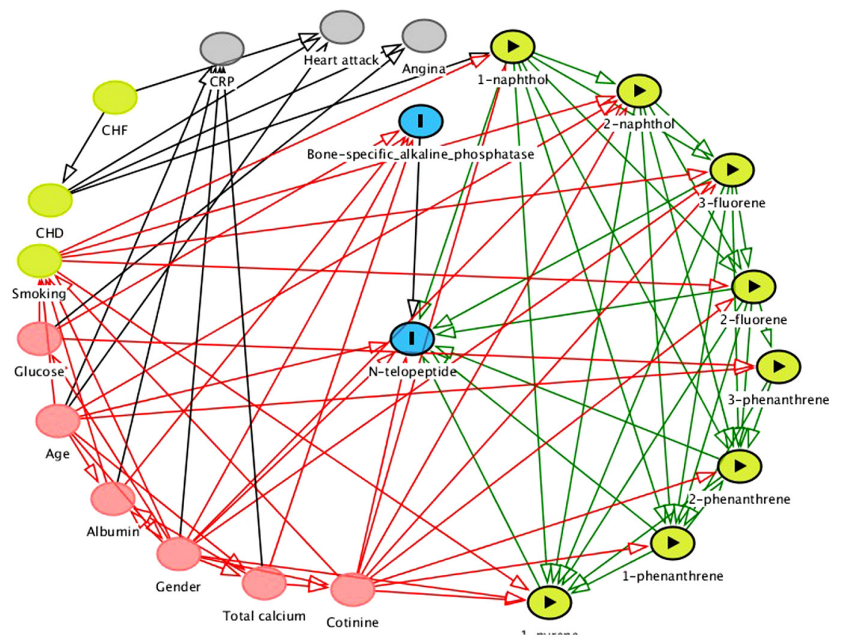

\section{Figure 1}

Directed Acyclic Graph. A full colour version of this figure is available at https://doi.org/10.1530/EJE-19-0750. regression model, which was adjusted for the following multivariate models. Model 1 included age, sex, and race/ethnicity; Model 2 included Model 1, cotinine, CRP, glucose, total calcium, and albumin levels; Model 3 included Model 2 and history of CHF, CHD, angina, heart attack, and cigarette use. We tested for effect modification by polycyclic aromatic hydrocarbon groups and gender by including interaction terms in the models for the N-telopeptide and bone-specific alkaline phosphatase. Because of the significant findings of the interaction testing, we performed further stratified analyses. A $P$ value $\leq 0.05$ was considered statistically significant. Differences in group characteristics were compared using the ANOVA and Bonferroni post hoc comparisons. All analyses in the current study were conducted using the Statistical Package for the Social Sciences software version 22.0 (SPSS Inc.) for Windows.

\section{Results}

The participants comprised 692 males and 716 females; their demographic and laboratory data are shown in Table 1 . The mean ages were $49.25 \pm 17.62$ years and $47.70 \pm 19.26$ years for males and females, respectively. The male participants had significantly higher levels of cotinine, glucose, total calcium, and albumin than female participants. In addition, a higher prevalence of cigarette use was observed in the male population than female population.

Table 2 displays the associations between polycyclic aromatic hydrocarbon metabolites as well as $\mathrm{N}$-telopeptide and bone-specific alkaline phosphatase. A positive relationship was observed between all polycyclic aromatic hydrocarbon metabolites, except 3-phenanthrene and N-telopeptide after adjustment of covariables (1-napthol: $\beta=0.006, \quad 95 \% \quad C I=0.004-0.008 ; 2$-napthol: $\beta=0.021$, 95\% CI $=0.017-0.025$; 3-fluorene: $\beta=0.431,95 \% \mathrm{CI}=$ 0.355-0.507; 2-fluorene: $\beta=0.168,95 \% \mathrm{CI}=0.134-0.202$; 1-phenanthrene: $\quad \beta=0.264, \quad 95 \% \quad C I=0.197-0.331$; 2-phenanthrene: $\beta=0.503,95 \% \mathrm{CI}=0.351-0.655$; and 1-pyrene: $\beta=0.157,95 \% \mathrm{CI}=0.083-0.230$ ). However, no significant association was observed between polycyclic aromatic hydrocarbon metabolites and bone-specific alkaline phosphatase.

Figures 2 and 3 depict the sex-based relationships between polycyclic aromatic hydrocarbon metabolites as well as N-telopeptide and bone-specific alkaline phosphatase. The positive relationship between polycyclic aromatic hydrocarbon metabolites and 
Table 1 Characteristics of study population.

\begin{tabular}{l}
\hline Variables \\
\hline Continuous variables, mean (S.D.) \\
Age (years) \\
Glucose $(\mathrm{mg} / \mathrm{dL})$ \\
Total calcium $(\mathrm{mg} / \mathrm{dL})$ \\
Albumin $(\mathrm{g} / \mathrm{dL})$ \\
C-reactive $\mathrm{protein}(\mathrm{mg} / \mathrm{dL})$ \\
Cotinine $(\mathrm{ng} / \mathrm{mL})$ \\
1-Naphthol $(\mu \mathrm{g} / \mathrm{L})$ \\
2-Naphthol $(\mu \mathrm{g} / \mathrm{L})$ \\
3-Fluorene $(\mu \mathrm{g} / \mathrm{L})$ \\
2-Fluorene $(\mu \mathrm{g} / \mathrm{L})$ \\
3-Phenanthrene $(\mu \mathrm{g} / \mathrm{L})$ \\
1-Phenanthrene $(\mu \mathrm{g} / \mathrm{L})$ \\
2-Phenanthrene $(\mu \mathrm{g} / \mathrm{L})$ \\
1-Pyrene $(\mu \mathrm{g} / \mathrm{L})$ \\
Category variables $(\%)$ \\
Congestive heart failure \\
Coronary heart disease \\
Angina/angina pectoris \\
Heart attack \\
Cancer or malignancy \\
Cigarette use
\end{tabular}

\begin{tabular}{c}
\hline Male \\
\hline $49.25(17.62)$ \\
$100.08(38.96)$ \\
$9.48(0.38)$ \\
$4.34(0.30)$ \\
$0.42(1.04)$ \\
$71.54(140.93)$ \\
$6.31(14.26)$ \\
$7.36(13.38)$ \\
$0.46(0.82)$ \\
$0.90(1.51)$ \\
$0.53(4.97)$ \\
$0.27(0.45)$ \\
$0.13(0.21)$ \\
$0.15(0.85)$ \\
$18(2.6)$ \\
$42(6.1)$ \\
$26(3.8)$ \\
$34(4.9)$ \\
$55(7.9)$ \\
$421(60.8)$
\end{tabular}

\begin{tabular}{|c|c|}
\hline Female & $P$ value \\
\hline $47.72(19.26)$ & 0.002 \\
\hline $93.48(26.81)$ & 0.004 \\
\hline $9.37(0.42)$ & 0.025 \\
\hline $4.09(0.34)$ & 0.013 \\
\hline $0.56(0.84)$ & 0.110 \\
\hline $36.92(102.38)$ & $<0.001$ \\
\hline $7.62(25.84)$ & 0.004 \\
\hline $5.84(10.45)$ & 0.003 \\
\hline $0.27(0.56)$ & $<0.001$ \\
\hline $0.59(1.28)$ & $<0.001$ \\
\hline $0.32(1.85)$ & 0.132 \\
\hline $0.26(0.80)$ & 0.645 \\
\hline $0.11(0.35)$ & 0.618 \\
\hline $0.08(0.17)$ & 0.010 \\
\hline $13(1.8)$ & 0.443 \\
\hline $23(3.2)$ & 0.776 \\
\hline $16(2.2)$ & 0.854 \\
\hline $17(2.4)$ & 0.279 \\
\hline $62(8.7)$ & 0.881 \\
\hline $251(35.1)$ & $<0.001$ \\
\hline
\end{tabular}

N-telopeptide was significantly observed in both sexes after adjustment of pertinent covariables (Fig. 2). Similarly, only 3-phenanthrene was unassociated with increased N-telopeptide level in both sexes. However, no significant association was observed between polycyclic aromatic hydrocarbon metabolites and bone-specific alkaline phosphatase in both sexes (Fig. 3).

Figures 4 and 5 depict the age-based associations between polycyclic aromatic hydrocarbon metabolites and bone turnover markers biomarker levels. All polycyclic aromatic hydrocarbon metabolites were significantly associated with increased N-telopeptide levels in participants aged $<65$ years $(P<0.001)$ (Fig. 4$)$. In contrast, all polycyclic aromatic hydrocarbon metabolites except 2-napthol and 3-phenanthrene showed positive relationships with N-telopeptide levels in participants $>65$ years. However, no significant association was observed between polycyclic aromatic hydrocarbon metabolites and bone-specific alkaline phosphatase in both age groups (Fig. 5).

\section{Discussion}

Urinary polycyclic aromatic hydrocarbon metabolites showed a significant association with increased N-telopeptide levels in nationally representative sample of U.S. adult population, independent of sex. This finding indicated that polycyclic aromatic hydrocarbon exposure induces the process of bone resorption, which causes bone turnover imbalance, leading to bone loss, even in osteoporosis. To the best of our knowledge, this study first examined the relationship between environmental polycyclic aromatic hydrocarbon exposure and bone turnover in a population-based cross-sectional survey.

In this study, polycyclic aromatic hydrocarbon metabolites were associated with increased N-telopeptide levels and not with bone-specific alkaline phosphatase; therefore, they affected bone resorption rather than bone formation. Several epidemiological studies suggest that cigarette smoking is a risk factor of decreased bone mass and osteoporosis. Smokers have significantly lower bone mineral density and higher risk of bone fracture than non-smokers $(18,19)$. Smoking can increase osteoclastic bone resorption. Increased levels of total and free deoxypyridinoline and C-telopeptide, which are bone resorption markers, are evident in current male smokers than previous smokers or non-smokers (20).

In addition, the levels of urinary N-telopeptide, which is another biomarker of bone resorption, is higher in elderly female smokers than in elderly female non-smokers (21). In a rat model study, Lee et al. demonstrated that benzo[a] pyrene, which is a polycyclic aromatic hydrocarbon found in cigarette, causes bone loss based on an in vivo study (14). Taken together, polycyclic aromatic hydrocarbons may have detrimental effects on bone turnover that 
Table 2 Association between PAHs and N-Telopeptides.

\begin{tabular}{l}
\hline Variables \\
\hline N-telopeptides \\
1-napthol \\
2-napthol \\
3-fluorene \\
2-fluorene \\
3-phenanthrene \\
1-phenanthrene \\
2-phenanthrene \\
1-pyrene \\
Bone alkaline phosphatase \\
1-napthol \\
2-napthol \\
3-fluorene \\
2-fluorene \\
3-phenanthrene \\
1-phenanthrene \\
2-phenanthrene \\
1-pyrene
\end{tabular}

\begin{tabular}{c}
\hline Model 1* \\
\hline$\beta^{\dagger}(95 \% \mathrm{Cl})$ \\
\hline $0.006(0.004,0.008)$ \\
$0.018(0.015,0.022)$ \\
$0.301(0.238,0.363)$ \\
$0.147(0.116,0.179)$ \\
$0.005(-0.007,0.017)$ \\
$0.269(0.202,0.336)$ \\
$0.518(0.366,0.669)$ \\
$0.162(0.088,0.235)$ \\
\\
$0.001(0.000,0.002)$ \\
$0.001(-0.001,0.003)$ \\
$0.012(-0.018,0.042)$ \\
$0.011(-0.004,0.025)$ \\
$-0.002(-0.007,0.004)$ \\
$0.028(-0.004,0.060)$ \\
$0.062(-0.009,0.134)$ \\
$0.012(-0.022,0.046)$ \\
\hline
\end{tabular}

$\begin{array}{r}\hline \text { P value } \\ \hline \\ <0.001 \\ <0.001 \\ <0.001 \\ <0.001 \\ 0.450 \\ <0.001 \\ <0.001 \\ <0.001 \\ \\ 0.173 \\ 0.221 \\ 0.428 \\ 0.165 \\ 0.533 \\ 0.085 \\ 0.085 \\ 0.481 \\ \hline\end{array}$

\begin{tabular}{c}
\hline Model 2* \\
\hline$\beta^{\dagger}(95 \% \mathrm{Cl})$ \\
\hline
\end{tabular}

$0.006(0.004,0.008)$

$0.021(0.017,0.025)$

$0.424(0.348,0.500)$

$0.168(0.134,0.201)$

$0.007(-0.005,0.019)$

$0.265(0.198,0.332)$

$0.503(0.351,0.655)$

$0.157(0.083,0.230)$
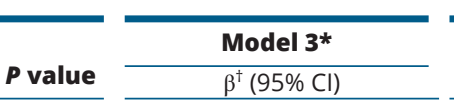

$P$ value

$<0.001$

$0.006(0.004,0.008)$

$<0.001$

$<0.001$

$<0.001$

$<0.001$

$0.021(0.017,0.025)$

$0.431(0.355,0.507)$

$<0.001$

0.270

$0.168(0.134,0.202)$

$<0.001$

$<0.001$

$0.007(-0.005,0.019) \quad 0.268$

$<0.001 \quad 0.264(0.197,0.331) \quad<0.001$

$<0.001 \quad 0.503(0.351,0.655) \quad<0.001$

$<0.001 \quad 0.157(0.083,0.230) \quad<0.001$

$0.001(0.000,0.002)$
$0.001(-0.001,0.003)$
$0.006(-0.030,0.042)$
$0.008(-0.008,0.024)$
$0.005(-0.010,0.001)$
$0.028(-0.004,0.059)$
$0.058(-0.013,0.129)$
$0.013(-0.021,0.047)$

0.193

$0.001(0.000,0.002)$

0.182

0.219

0.749

$0.001(-0.001,0.003)$

0.203

0.320

$0.008(-0.028,0.045)$

0.648

0.107

$0.008(-0.008,0.025)$

0.300

0.084

0.107

$-0.005(-0.010,0.001)$

0.112

$0.028(-0.004,0.059) \quad 0.085$

$0.059(-0.012,0.130)-0.102$

0.441

$0.014(-0.020,0.048)$

0.431

*Adjusted covariates: Model 1: age, gender, race/ethnicity; Model 2: Model 1 + glucose, total calcium, albumin, C-reactive protein, cotinine; Model 3: Model 2 + history of CHF, CHD, angina, heart attack, cancer, or malignancy, ever smoking; ${ }^{\dagger}$ indicates statistical significance

induced bone resorption via potential mechanisms. This result is consistent with our findings; polycyclic aromatic hydrocarbon metabolites were significantly associated with increased levels of N-telopeptide, which reflects the rate of bone resorption.

The physiological mechanisms of polycyclic aromatic hydrocarbon mediated by the aryl hydrocarbon receptor $(\mathrm{AhR})$ are well established $(22,23)$. This receptor is AhR, a dioxin receptor that is responsible for the toxicity of polyhalogenated hydrocarbons (24). It is a ligandactivated transcription factor, which exists in almost all human organs, such as the heart and liver, and tissues, including those of the ectoderm, muscle, and bone (25, $26,27)$. In a previous study, AhR was assumed to play an important role in bone homeostasis maintenance (28). Iqbal et al. demonstrated that osteoclastic bone resorption is induced by the interaction between polycyclic aromatic hydrocarbon and AhR via the activation of cytochrome P450 enzymes (29). These chemicals accelerated osteoclast formation in bone cells with benzo[a]pyrene-stimulated bone resorption and osteoclastogenesis in vivo (29). Accordingly, we speculated that other polycyclic aromatic hydrocarbons analyzed in this study have contributed to bone resorption via the similar pathways.

The activity of osteoblasts and osteoclasts decreases in bone tissues of elderly individuals (30). Becerikli et al. demonstrated that the levels of RANKL and NFATc1, which are markers of osteoclastogenesis, are significantly lower in older adults (31). Because of reduced bone resorption activity, polycyclic aromatic hydrocarbon metabolites may have a weak impact on N-telopeptide levels of elderly individuals.

The present study has some notable limitations. First, it has a cross-sectional design; therefore, the causal relationships between polycyclic aromatic hydrocarbon and N-telopeptide were not assessed. A long-term observation period should be considered in future studies. Second, N-telopeptide levels were measured in the urine sample, but the time sample collection was not considered; however, it is critical due to significant circadian variations. Patients in previous studies were instructed to fast to reduce these variations $(32,33)$. Fasting morning samples must be collected for optimal clinical examination. Third, urine samples instead of serum samples were used to assess the biomarker levels of bone resorption in this study. Serum biomarkers have better reproducibility and prevent correcting creatinine levels, which can cause variability in the analysis. In addition, the assessment of C-terminal telopeptides in serum samples using an antibody-specific immunoassay is an accurate method for predicting bone loss and fracture risk (34). An important study strength is that all multivariate statistical models were adjusted for cigarette use to identify the underlying mechanisms in smokers. Moreover, the generalizability of our findings may not be limited to adult populations in the United States. 
A 1-napthol 2-napthol 3-fluorene 2-fluorene

3-phen

1-phen

2-phen

1-pyrene

1-napthol 2-napthol

呈

2-fluorene-

3-phen

1-phen

2-phen

1-pyrene

1-napthol

2-napthol

3 -fluorene

2-fluorene

3-phen

1-phen

2-phen

1-pyrene
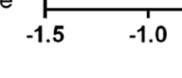

beta coefficients (NTx)

B

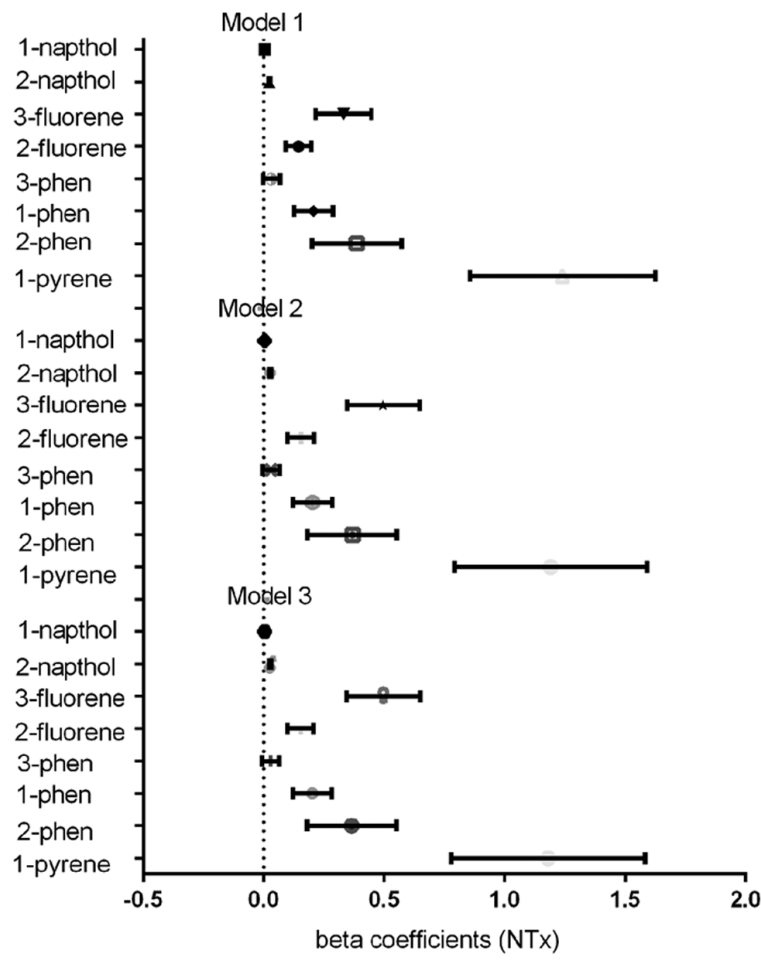

Figure 2

(A) Association between PAH metabolites and NTx in males. (B) Association between PAH metabolites and NTx in females.

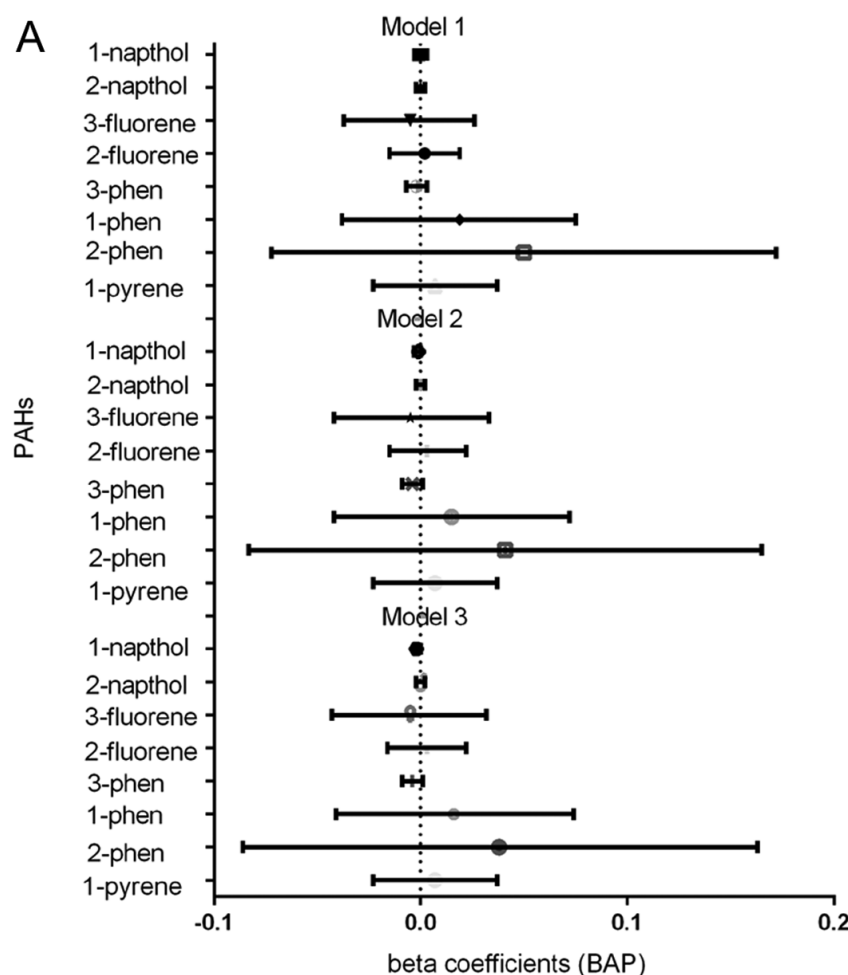

B 1-napthol 2-napthol 3-fluorene 2-fluorene 3-phen 1-phen 2-phen

1-pyrene

1-napthol

옴

2-napthol

2-fluorene

3-phen

1-phen

2-phen

1-pyrene

1-napthol

2-napthol

3-fluorene

2-fluorene

3-phen

1-phen

2-phen

1-pyrene

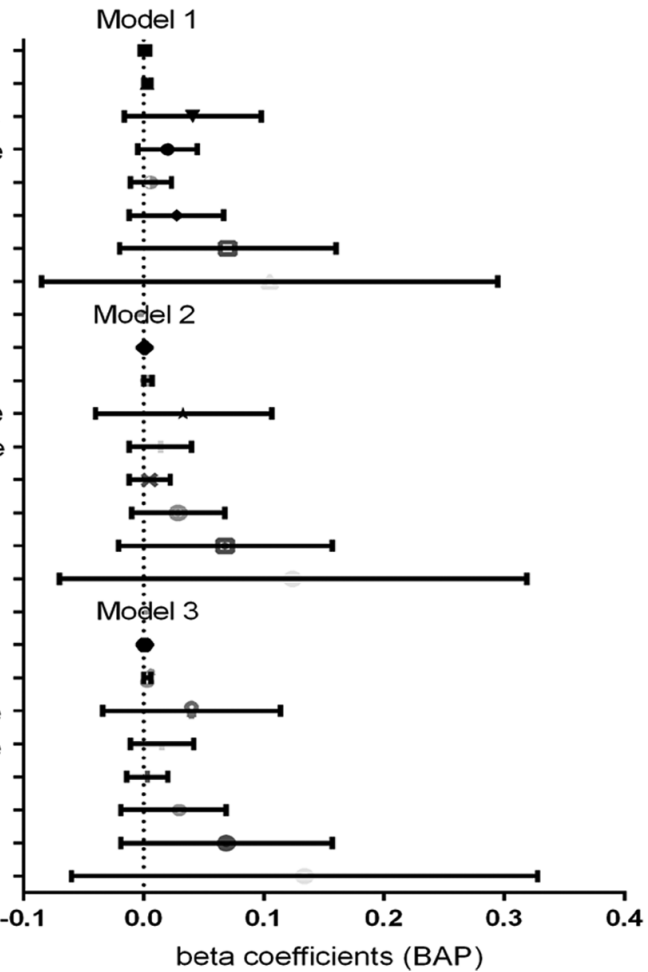

Figure 3

(A) Association between PAH metabolites and BAP in males. (B) Association between $\mathrm{PAH}$ metabolites and BAP in females. 

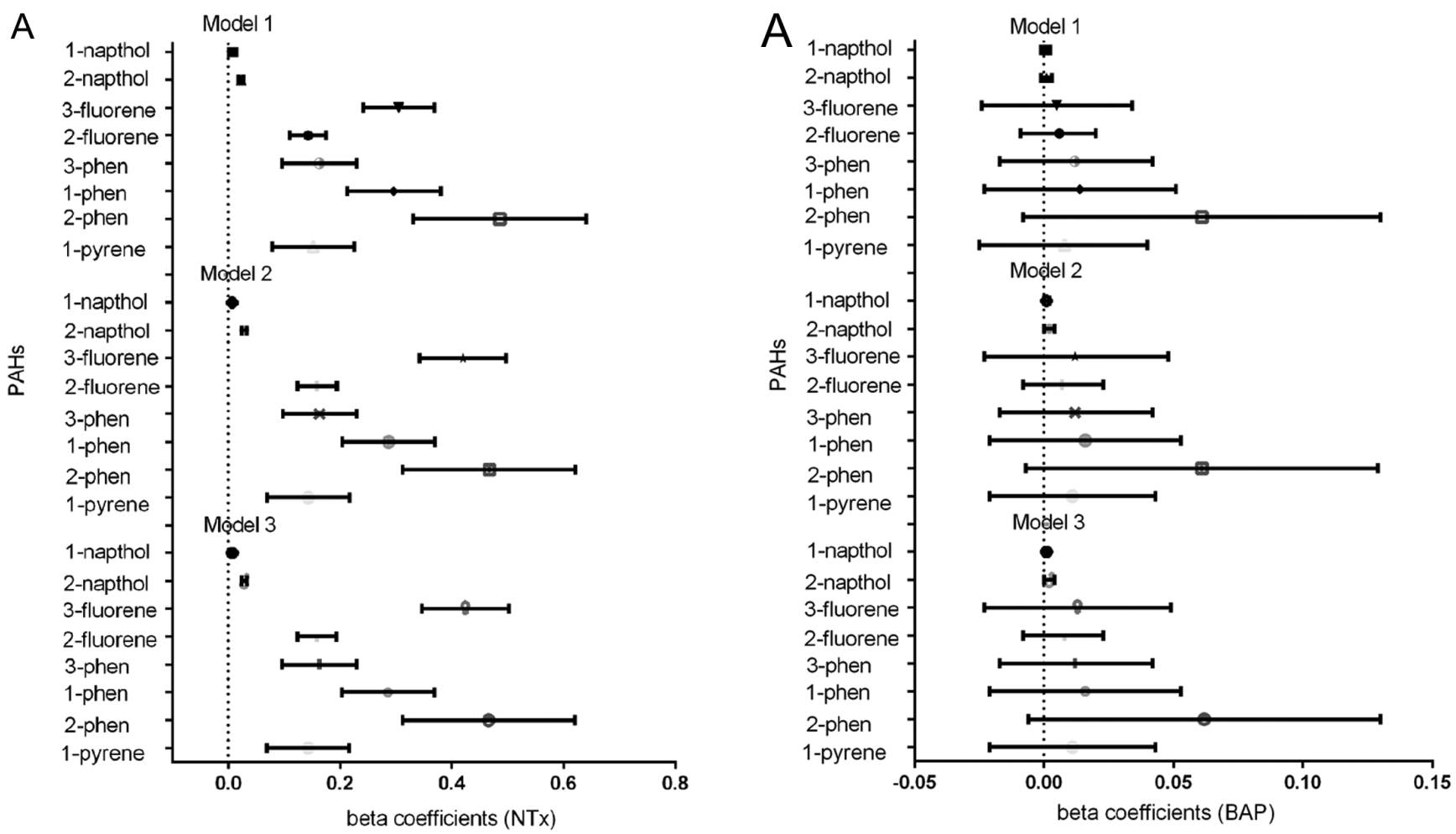

B

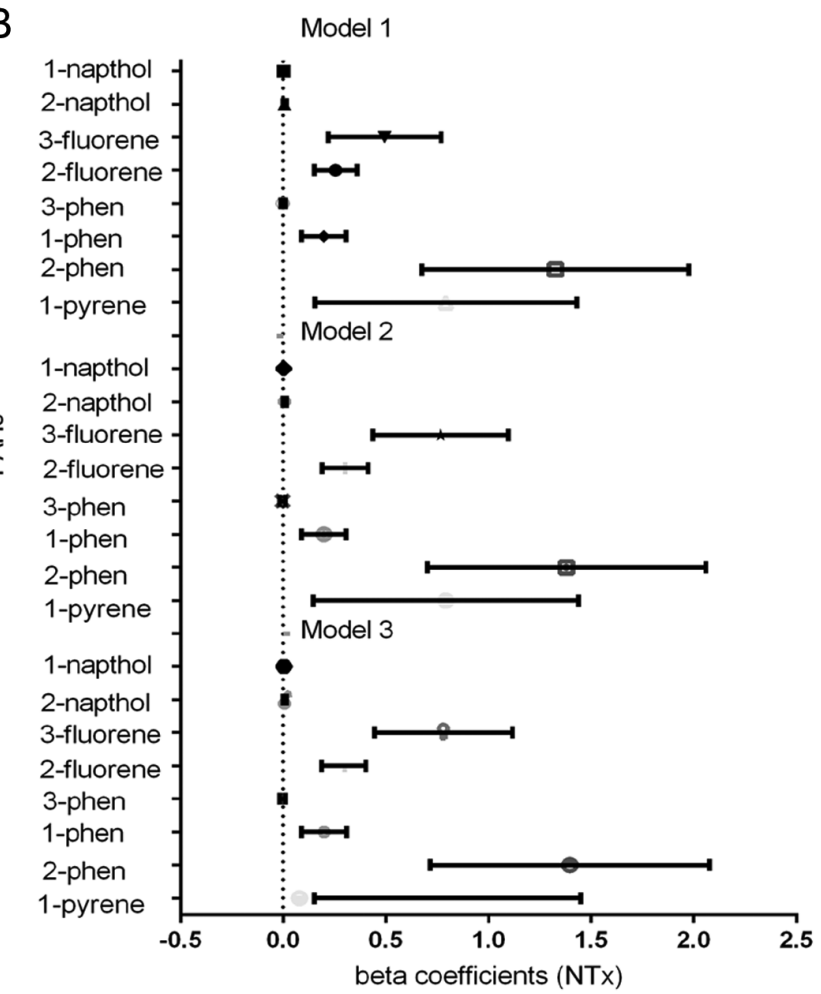

\section{Figure 4}

(A) Association between PAH metabolites and NTx in $<65$ years old. (B) Association between PAH metabolites and NTx in $>65$ years old.
$B$

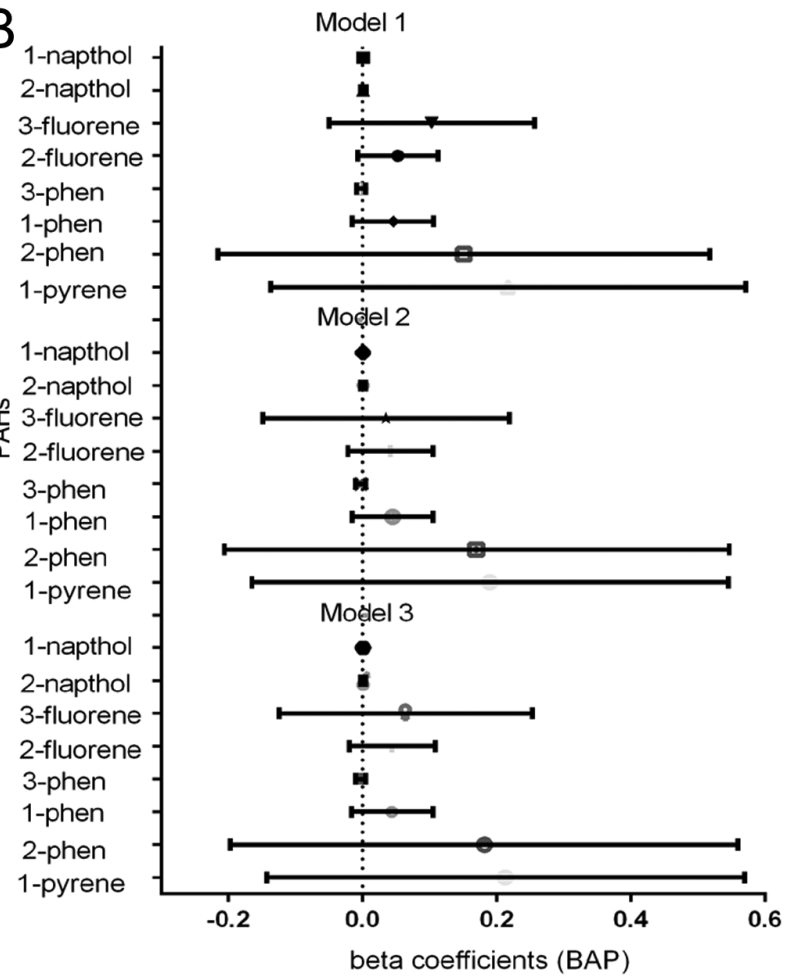

Figure 5

(A) Association between PAH metabolites and BAP in $<65$ years old. (B) Association between PAH metabolites and BAP in $>65$ years old. 


\section{Conclusion}

Our study highlighted the important role of polycyclic aromatic hydrocarbon exposure in the process of bone resorption. Urinary polycyclic aromatic hydrocarbon metabolites were positively associated with increased levels of N-telopeptide, independent of sex. In addition, all polycyclic aromatic hydrocarbon metabolites were significantly correlated with N-telopeptide in participants aged $<60$ years. The polycyclic aromatic hydrocarbon exposure was found to have a detrimental effect on health, particularly carcinogenic risks. However, its impact on bone loss has not been fully elucidated. Compared with N-telopeptide, bone-specific alkaline phosphatase was not significantly associated with polycyclic aromatic hydrocarbon metabolites. Thus, polycyclic aromatic hydrocarbon metabolites may be involved in the progression of bone resorption. Moreover, future studies exploring the mechanisms underlying the effects of polycyclic aromatic hydrocarbon metabolites on bone remodeling process must be conducted. To the best of our knowledge, this epidemiological study first presented the association between polycyclic aromatic hydrocarbon, a widely distributed environmental pollutant, and bone loss that may result in osteoporosis and fracture.

\section{Declaration of interest}

The authors declare that there is no conflict of interest that could be perceived as prejudicing the impartiality of this study.

\section{Funding}

This research did not receive any specific grant from any funding agency in the public, commercial or not-for-profit sector.

\section{Author contribution statement}

Yuan-Yuei Chen contributed to the design of the study, was responsible for the management and retrieval of data, contributed to initial data analysis and interpretation, and drafted the initial manuscript. Yuan-Yuei Chen, Tung-Wei Kao, Chung-Ching Wang, Chen-Jung Wu, Yi-Chao Zhou, and WeiLiang Chen decided upon the data collection methods. Yuan-Yuei Chen and Wei-Liang Chen were also responsible for the data analysis decisions. Wei-Liang Chen conceptualized and designed the study, supervised all aspects of the study, critically reviewed and revised the manuscript, and approved the final manuscript as submitted. All authors meet the ICMJE criteria for authorship.

\section{Acknowledgements}

All or part of the data used in this research were authorized by and received from the National Health and Nutrition Examination Survey. Any interpretation or conclusion described in this paper does not represent the views of the National Health and Nutrition Examination Survey.

\section{References}

1 Fetzer JC. The chemistry and analysis of large PAHs. Polycyclic Aromatic Compounds 200727 143-162. (https://doi. org/10.1080/10406630701268255)

2 Abdel-Shafy HI \& Mansour MSM. A review on polycyclic aromatic hydrocarbons: source, environmental impact, effect on human health and remediation. Egyptian Journal of Petroleum 201625 107-123. (https://doi.org/10.1016/j.ejpe.2015.03.011)

3 Armstrong B, Hutchinson E, Unwin J \& Fletcher T. Lung cancer risk after exposure to polycyclic aromatic hydrocarbons: a review and meta-analysis. Environmental Health Perspectives 2004112 970-978. (https://doi.org/10.1289/ehp.6895)

4 Mastrangelo G, Fadda E \& Marzia V. Polycyclic aromatic hydrocarbons and cancer in man. Environmental Health Perspectives 1996104 1166-1170. (https://doi.org/10.1289/ehp.961041166)

5 Burstyn I, Boffetta P, Heederik D, Partanen T, Kromhout H, Svane O, Langard S, Frentzel-Beyme R, Kauppinen T, Stucker I et al. Mortality from obstructive lung diseases and exposure to polycyclic aromatic hydrocarbons among asphalt workers. American Journal of Epidemiology 2003158 468-478. (https://doi.org/10.1093/aje/ kwg180)

6 Hu H, Kan H, Kearney GD \& Xu X. Associations between exposure to polycyclic aromatic hydrocarbons and glucose homeostasis as well as metabolic syndrome in nondiabetic adults. Science of the Total Environment 2015505 56-64. (https://doi.org/10.1016/j. scitotenv.2014.09.085)

7 Xu X, Cook RL, Ilacqua VA, Kan H, Talbott EO \& Kearney G. Studying associations between urinary metabolites of polycyclic aromatic hydrocarbons (PAHs) and cardiovascular diseases in the United States. Science of the Total Environment 2010408 4943-4948. (https://doi.org/10.1016/j.scitotenv.2010.07.034)

8 Korashy HM \& El-Kadi AO. The role of aryl hydrocarbon receptor in the pathogenesis of cardiovascular diseases. Drug Metabolism Reviews 200638 411-450. (https://doi. org/10.1080/03602530600632063)

9 Boskey AL \& Coleman R. Aging and bone. Journal of Dental Research 201089 1333-1348. (https://doi.org/10.1177/0022034510377791)

10 Sims NA \& Martin TJ. Coupling the activities of bone formation and resorption: a multitude of signals within the basic multicellular unit. BoneKEy Reports 20143 481. (https://doi.org/10.1038/ bonekey.2013.215)

11 Krall EA \& Dawson-Hughes B. Smoking increases bone loss and decreases intestinal calcium absorption. Journal of Bone and Mineral Research 199914 215-220. (https://doi.org/10.1359/ jbmr.1999.14.2.215)

12 Patel RA, Wilson RF, Patel PA \& Palmer RM. The effect of smoking on bone healing: a systematic review. Bone and Joint Research 20132 102-111. (https://doi.org/10.1302/2046-3758.26.2000142)

13 Kanis JA, Johnell O, Oden A, Johansson H, De Laet C, Eisman JA, Fujiwara S, Kroger H, McCloskey EV, Mellstrom D et al. Smoking and fracture risk: a meta-analysis. Osteoporosis International 200516 155-162. (https://doi.org/10.1007/s00198-004-1640-3)

14 Lee LL, Lee JS, Waldman SD, Casper RF \& Grynpas MD. Polycyclic aromatic hydrocarbons present in cigarette smoke cause bone loss in an ovariectomized rat model. Bone 200230 917-923. (https://doi. org/10.1016/s8756-3282(02)00726-3)

15 Rosen HN, Moses AC, Garber J, Iloputaife ID, Ross DS, Lee SL \& Greenspan SL. Serum CTX: a new marker of bone resorption that shows treatment effect more often than other markers because of low coefficient of variability and large changes with bisphosphonate therapy. Calcified Tissue International 200066 100-103. (https://doi. org $/ 10.1007 / \mathrm{pl} 100005830$ )

16 Iba K, Takada J, Hatakeyama N, Ozasa Y, Wada T \& Yamashita T. Changes in urinary NTX levels in patients with primary osteoporosis 
undergoing long-term bisphosphonate treatment. Journal of Orthopaedic Science 200813 438-441. (https://doi.org/10.1007/ s00776-008-1265-z)

17 (CDC) CfDCaP. National Health and Examination Survey. Data Documentation, Codebook, and Frequencies. Phthalates, Phytoestrogens, and PAHs-Urine 2012.

18 Law MR \& Hackshaw AK. A meta-analysis of cigarette smoking, bone mineral density and risk of hip fracture: recognition of a major effect. BMJ 1997315 841-846. (https://doi.org/10.1136/ bmj.315.7112.841)

19 Yoon V, Maalouf NM \& Sakhaee K. The effects of smoking on bone metabolism. Osteoporosis International 201223 2081-2092. (https:// doi.org/10.1007/s00198-012-1940-y)

20 Szulc P, Garnero P, Claustrat B, Marchand F, Duboeuf F \& Delmas PD. Increased bone resorption in moderate smokers with low body weight: the Minos study. Journal of Clinical Endocrinology and Metabolism 2002 87 666-674. (https://doi.org/10.1210/jcem.87.2.8232)

21 Rapuri PB, Gallagher JC, Balhorn KE \& Ryschon KL. Smoking and bone metabolism in elderly women. Bone 200027 429-436. (https:// doi.org/10.1016/s8756-3282(00)00341-0)

22 Marlowe JL \& Puga A. Aryl hydrocarbon receptor, cell cycle regulation, toxicity, and tumorigenesis. Journal of Cellular Biochemistry 200596 1174-1184. (https://doi.org/10.1002/ jcb.20656)

23 Nebert DW, Dalton TP, Okey AB \& Gonzalez FJ. Role of aryl hydrocarbon receptor-mediated induction of the CYP1 enzymes in environmental toxicity and cancer. Journal of Biological Chemistry 2004279 23847-23850. (https://doi.org/10.1074/jbc.R400004200)

24 Fujii-Kuriyama Y \& Mimura J. Molecular mechanisms of AhR functions in the regulation of cytochrome P450 genes. Biochemical and Biophysical Research Communications 2005338 311-317. (https:// doi.org/10.1016/j.bbrc.2005.08.162)

25 Rowlands JC \& Gustafsson JA. Aryl hydrocarbon receptor-mediated signal transduction. Critical Reviews in Toxicology 199727 109-134. (https://doi.org/10.3109/10408449709021615)
26 Tsay JJ, Tchou-Wong KM, Greenberg AK, Pass H \& Rom WN. Aryl hydrocarbon receptor and lung cancer. Anticancer Research 201333 1247-1256.

27 Herlin M, Finnila MA, Zioupos P, Aula A, Risteli J, Miettinen HM, Jamsa T, Tuukkanen J, Korkalainen M, Hakansson $\mathrm{H}$ et al. New insights to the role of aryl hydrocarbon receptor in bone phenotype and in dioxin-induced modulation of bone microarchitecture and material properties. Toxicology and Applied Pharmacology 2013273 219-226. (https://doi.org/10.1016/j.taap.2013.09.002)

28 Yu TY, Kondo T, Matsumoto T, Fujii-Kuriyama Y \& Imai Y. Aryl hydrocarbon receptor catabolic activity in bone metabolism is osteoclast dependent in vivo. Biochemical and Biophysical Research Communications 2014450 416-422. (https://doi.org/10.1016/j.bbrc.2014.05.114)

29 Iqbal J, Sun L, Cao J, Yuen T, Lu P, Bab I, Leu NA, Srinivasan S, Wagage S, Hunter CA et al. Smoke carcinogens cause bone loss through the aryl hydrocarbon receptor and induction of Cyp1 enzymes. PNAS 2013110 11115-11120. (https://doi.org/10.1073/ pnas.1220919110)

30 Shen L, Zhou S \& Glowacki J. Effects of age and gender on WNT gene expression in human bone marrow stromal cells. Journal of Cellular Biochemistry 2009106 337-343. (https://doi.org/10.1002/jcb.22010)

31 Becerikli M, Jaurich H, Schira J, Schulte M, Döbele C, Wallner C, Abraham S, Wagner JM, Dadras M, Kneser U et al. Age-dependent alterations in osteoblast and osteoclast activity in human cancellous bone. Journal of Cellular and Molecular Medicine 201721 2773-2781. (https://doi.org/10.1111/jcmm.13192)

32 Christgau S. Circadian variation in serum CrossLaps concentration is reduced in fasting individuals. Clinical Chemistry 200046431.

33 Ju HS, Leung S, Brown B, Stringer MA, Leigh S, Scherrer C, Shepard K, Jenkins D, Knudsen J \& Cannon R. Comparison of analytical performance and biological variability of three bone resorption assays. Clinical Chemistry 199743 1570-1576.

34 Garnero P, Borel O \& Delmas PD. Evaluation of a fully automated serum assay for C-terminal cross-linking telopeptide of type I collagen in osteoporosis. Clinical Chemistry 200147 694-702.

Received 22 September 2019

Revised version received 12 November 2019

Accepted 10 December 2019 